\title{
IMPLEMENTATION OF A CENTRAL-LINE CARE BUNDLE IN A TERTIARY NEONATAL INTENSIVE CARE UNIT
}

Stefanie van der Kruit, Maarten Rijpert, Jessica van Veen, Anne De Jaegere, Joke Wielenga

Neonatal Intensive Care Unit, Emma Children's Hospital, Amsterdam university medical centers, location AMC, The Netherlands, s.vanderkruit@amc.nl

\section{Background and aims}

Central-line-associated bloodstream infections (CLABSIs) are a major problem in neonatal intensive care units (NICUs) worldwide. The average incidence of CLABSIs on a NICU is 8.4 (IQR 3.7-16) per 1000 catheter days and the overall mortality of children with a CLABSI is $4-20 \%$. There is increasing scientific evidence that the implementation of a central-line care bundle (defined as a combination of interventions to prevent CLABSI) reduces the incidence of CLABSI up to $65 \%$. We aimed to compose and implement a central-line care bundle in our NICU.

\section{Methods}

We composed a multidisciplinary central-line care bundle based on (inter)national guidelines and existing central-line care bundles. Bundle elements were adapted for local applicability where necessary and were implemented all in once after extensive training and learning sessions. Implementation of bundle elements was evaluated through checklists, questionnaires and multidisciplinary reflection meetings.

\section{Results}

Our NICU's central-line care bundle consists of a strict protocol of measures regarding the preparation (e.g. gathering required materials, preoperative hand disinfection, closure of the intensive care unit), insertion (e.g. proper disinfection, using double glove technique, minimal catheter handling, reporting in electronic chart) and maintenance (e.g. catheter maintenance checklist) of a central-line. Evaluation of implementation showed an increase in hygiene compliance and awareness.

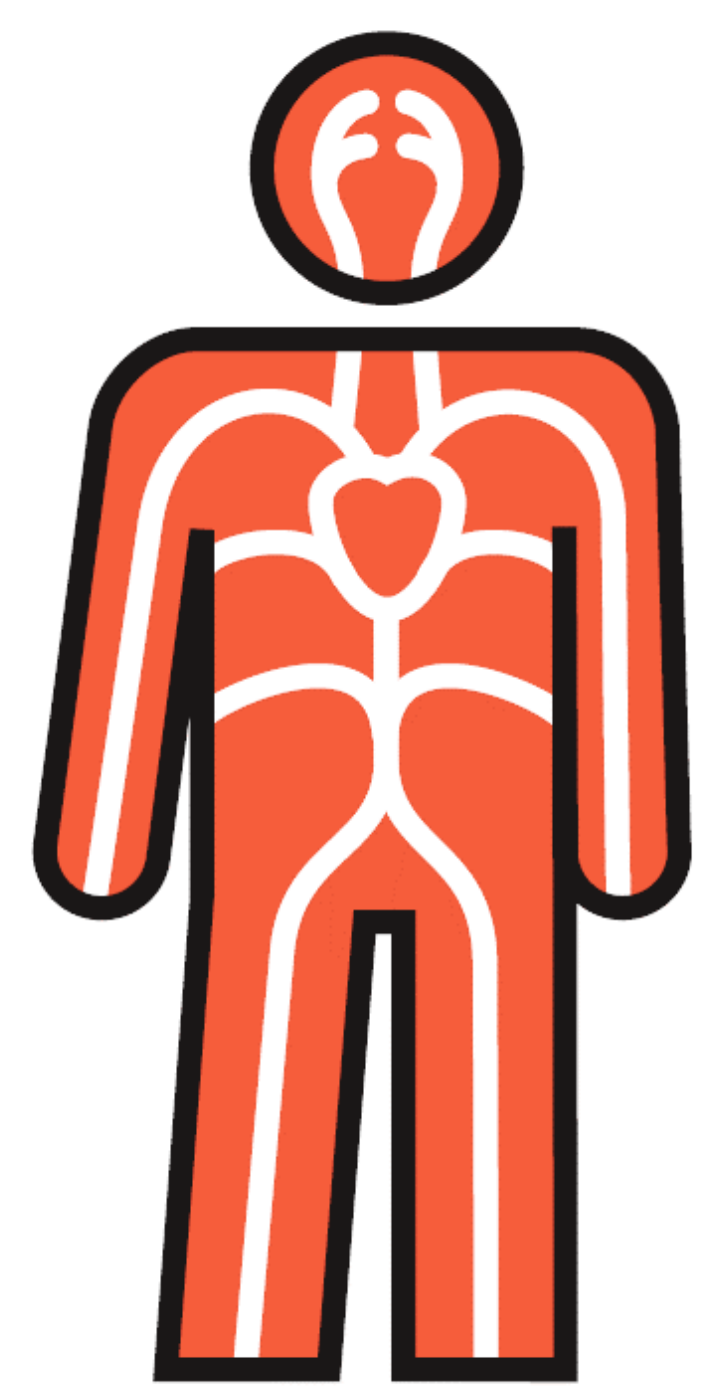

Source: www.vmszorg.nl

\section{Conclusions}

By composing and implementing a multidisciplinary central-line care bundle in our NICU, the hygiene awareness during preparation, insertion and maintenance of a central-line has increased considerably. Possible reduction of CLABSI rate following the implementation of our central-line care bundle has yet to be determined. 